\title{
Las Relaciones Públicas y la gestión de la comunicación. Caso del Instituto del Mar del Perú (IMARPE)
}

\author{
Recibido: 18 de enero de 2015 \\ Aceptado: 01 de julio de 2016 \\ Publicado: 30 de noviembre de 2016
}

Patricia Bobadilla Terán

pbobadillat@usmp.pe

Universidad de San Martín de Porres (Perú)

Resumen: En la actualidad, las Relaciones Públicas (RR. PP.) están tomando mayor protagonismo en la escena de las organizaciones públicas, son llamadas a ser las gestoras de los diferentes procesos de comunicación ya sea para mejorar la imagen de la entidad, generar mayor identidad en sus colaboradores o para mejorar la calidad de relaciones de la organización con sus públicos. El objetivo de la presente investigación es establecer la relación que existe entre las RR. PP. y la gestión de la comunicación (GC) en el Instituto del Mar del Perú (IMARPE), concebida las RR. PP. como la disciplina encargada de velar por la imagen, la identidad organizacional y la GC, así como la administración de los diferentes flujos y canales de la comunicación que puede presentar la organización.

El método de la investigación es descriptivo, correlacional, explicativo de tipo cuantitativo y no experimental. La muestra evaluada fue de 196 colaboradores de una población de 520 trabajadores a nivel nacional del IMARPE. Los resultados del trabajo demostraron la significativa relación que existe entre las RR. PP. y la GC y cómo la correcta aplicación de técnicas y herramientas van a favorecer la comunicación organizacional en la institución.

Palabras clave: Relaciones Públicas, gestión de la comunicación, imagen, identidad, flujos de la comunicación, canales de la comunicación.

Abstract: These days, Public Relations are getting more important prominence in public organizations; they are destined to be the agent of different communication process, either to improve organization image or to get better relations between the organization and its publics. The objective of this research is establishing a relation that exists between the Public Relations and the Communication Management in Instituto del Mar del Peru 
(IMARPE); Public Relations being conceived as discipline in charge of look out the organizational image and identify, and Communication Management as the administration of different flows and communication channels which could have the organization.

The investigation method is descriptive, correlative, explanatory; quantitative non experimental type. The evaluated sample was of 196 coworkers of 520 coworker 's population, speaking of national coverage of IMARPE. The results of this work demonstrated the significant relation that exists among Public Relations and Communication Management, and how the correct application of communication techniques and tools can advantage the organizational communication in the institution.

Key words: Public Relations, Communication Management, Image, Identity, Communication Flows, Communication Channels.

\section{Introducción}

En la actualidad, las organizaciones están tomando en cuenta la GC como herramienta para el logro de sus objetivos empresariales, así como también el fortalecimiento de las relaciones institucionales y el mantenimiento de un adecuado clima laboral. Es así que Strauk y Guillén (2014: 153) afirman que "la concepción estratégica de la comunicación es un planteamiento en constante evolución, que de acuerdo al tipo de organización adquiere matices particulares". Ya que no podemos hablar de un solo modelo de comunicación para todas las organizaciones, hay que reconocer las características y alcances de cada una.

En nuestro país aún existe desconocimiento de la importancia de la GC y del rol del profesional de RR. PP. dentro de la organización. Las diversas instituciones estatales vienen realizando tácticas para mejorar el nivel de comunicación con el público en general, basando estos esfuerzos en la publicidad y el marketing, es decir, una comunicación unidireccional con emisión de mensajes que buscan la aceptación de la comunidad:

"La comunicación en instituciones del Estado debe basarse en relaciones de 'comprensión mutua' entre gobierno y ciudadanos, contexto en el cual estos últimos deben participar activamente, teniendo en cuenta el valor y la importancia que representa la existencia de un diálogo continuo entre los ciudadanos y sus instituciones" (ibídem: 156).

Tras lo expuesto anteriormente, concebimos la GC en las organizaciones como un factor que garantiza la consecución de objetivos organizacionales y el mantenimiento de buenas relaciones entre la organización y sus públicos estratégicos. La institución estudiada, IMARPE, es un organismo técnico especializado adscrito al Ministerio de la Producción (PRODUCE), con una labor trascendente para la economía del país, puesto que se encarga del monitoreo y evaluación de los diferentes recursos marinos, información relevante para el sector pesquero, el mismo que representa el 7\% del aporte total de divisas para el Perú a través de sus exportaciones de aceite y harina de pescado. Asimismo, representa el $2 \%$ del PBI total nacional y genera anualmente empleos para 221 mil personas. 
El objetivo de este artículo es mostrar la importancia que tiene la GC en IMARPE para el mejoramiento de las relaciones entre su sede central y sus laboratorios descentralizados. Además, se examinan los métodos aplicados de las RR. PP. y su influencia en el fortalecimiento de la identidad de los miembros de la organización y, por ende, en una mejor proyección de imagen. De esta forma, se realiza un diagnóstico del proceso de comunicación dentro de la organización y cómo éste interviene en el quehacer de la Oficina de Comunicaciones e Imagen Institucional de IMARPE.

\section{Desarrollo de las RR. PP. y la GC}

Wilcox, Cameron y Xifra (2006: 7) sostienen que esta disciplina comunicacional engloba "una función directiva independiente, que permite establecer y mantener líneas de comunicación, comprensión, aceptación y cooperación mutua entre una organización y sus públicos; implica la resolución de problemas; ayuda a los directivos a estar informados y poder reaccionar ante la opinión pública; define y destaca la responsabilidad de los directivos que deben servir al interés público; ayuda a la dirección a mantenerse al día y a utilizar los cambios de forma efectiva, sirviendo como un sistema de alerta para ayudar a anticipar las tendencias; utiliza la investigación y técnicas de comunicación éticas como principales herramientas".

Enmarcado en su función directiva (ibídem: 8), las RR. PP. trabajan, evalúan y mejoran las relaciones que presenta la organización con sus públicos, convirtiéndose en eje estratégico de esta. No sólo toma en consideración la comunicación en sí (que se configura como el máximo instrumento orientado a la consolidación de la reputación empresarial) sino también a los públicos que serán su objeto de estudio, campo de acción y su fin supremo, a fin de establecer lazos mutuamente beneficiosos.

A lo largo de su evolución histórica, se identifican tres escuelas: la norteamericana se caracterizó inicialmente por el uso de técnicas de promoción, convirtiendo al relacionista como un "agente de imagen". La europea se centró en la motivación y desempeño del "factor humano", buscando fortalecer los vínculos de confianza, solidaridad e identificación de los stakeholders con la organización. Por último, la latinoamericana recoge los aportes de sus predecesoras y trata de compatibilizar los intereses de la empresa con los de la sociedad, urgiendo el despliegue de programas de responsabilidad social a fin de paliar la ausencia del Estado en muchos puntos de la región

Ante la necesidad de contar con profesionales que comprendan tanto el clima interno de la organización como la naturaleza de la región latinoamericana, surge el requerimiento de cederle a la comunicación un carácter más estratégico dentro de la entidad. Así, Losada (2004: 49) explica que la GC es "una función esencial, al igual que otras áreas directivas de la organización, y había que someterla a una planificación sistemática”. Esta perspectiva estratégica obliga a ir más allá de la mera emisión de mensajes, sino de generar retroalimentación con los públicos de interés. 


\section{El Instituto del Mar del Perú (IMARPE)}

Con más de cincuenta años de vida institucional ${ }^{1}$, es un organismo técnico especializado adscrito al Ministerio de la Producción (PRODUCE), cuya misión ${ }^{2}$ es asesorar al Estado para la toma de decisiones referidas al ordenamiento y regulación de las pesquerías y de la acuicultura, respetando y promoviendo los conceptos de desarrollo sustentable, conservación de la biodiversidad marina, protección del medio ambiente y pesca responsable ${ }^{3}$. Posee con una sede central ubicada en el Callo y diez laboratorios descentralizados ${ }^{4}$ situados a lo largo del litoral peruano ${ }^{5}$. Debido a la riqueza y variedad del mar peruano y su ecosistema, IMARPE cuenta con cinco direcciones generales de investigación ${ }^{6}$ y una flota especial que materializa dicha labor ${ }^{7}$.

El Consejo Directivo es el órgano rector, encargado de establecer los objetivos y políticas de la institución. Está integrado por siete personas: presidente ${ }^{8}$, director ejecutivo, un representante de la $\mathrm{DHN}$, un representante de las universidades peruanas y tres científicos y/o profesionales con especialidad afín a la actividad del Instituto. En la actualidad, este instituto tiene 520 colaboradores a nivel nacional, dividiéndose esto en 345 en Lima y 175 en los laboratorios descentralizados.

La Oficina de Comunicaciones e Imagen Institucional de IMARPE (adscrita a la Secretaría General) cuenta con tres comunicadores de distintas especialidades: RR. PP., diseño gráfico y audiovisuales. Su plan de comunicación interna contiene cinco objetivos y cuatro estrategias que se detallan a continuación (figura 1):

1 IMARPE tiene su génesis cuando se establece el Instituto de Investigaciones de Recursos Marinos (IREMAR), cuyas actividades se inician en 1960. Tres años después se crea IMARPE bajo Decreto Supremo N 021.

2 Tal como aparece en su portal oficial, la visión de IMARPE (2016) busca "mantener el liderazgo en la investigación científica y tecnológica de los ecosistemas acuáticos, la biodiversidad, la acuicultura y sus pesquerías, asesorando al Ministerio de la Producción en el ordenamiento pesquero y conservación del ambiente marino, en tiempo real y en el marco de un enfoque ecosistémico, utilizando tecnología de punta para la generación de actividades productivas". Presenta 4 valores institucionales: transparencia, veracidad, confiabilidad y excelencia.

3 Cabe destacar que IMARPE preside el Comité Multisectorial encargado del Estudio Nacional del Fenómeno "El Niño" (ENFEN), junto con otras instituciones como el Servicio Nacional de Meteorología e Hidrología del Perú (SENAMHI), la Dirección de Hidrografía y Navegación de la Marina de Guerra del Perú (DHN), el Instituto Geofísico del Perú (IGP), la Autoridad Nacional de Agua (ANA) y el Instituto Nacional de Defensa Civil (INDECI).

4 Se ubican en Tumbes, Paita (Piura), Santa Rosa (Lambayeque), Huanchaco (La Libertad), Chimbote (Áncash), Huacho (Lima), Pisco (Ica), Camaná (Arequipa), Puno e Ilo (Moquegua).

5 El laboratorio de Puno no se encuentra en la costa: se dedica a la investigación y monitoreo del Lago Titicaca.

6 La dirección general de investigaciones oceanográficas y cambio climático; en acuicultura; en hidroacústica, sensoramiento remoto y artes de pesca; en recursos pelágicos; recursos demersales y litorales.

7 Los buques BIC Humboldt, el BIC José Olaya Balandra y el BIC Luis Flores Portugal; tres embarcaciones multipropósito de menor escala (IMARPE IV, IMARPE V e IMARPE VI) y otras asignadas para apoyo.

8 Oficial vicealmirante o contralmirante en situación de retiro de la Marina de Guerra. 
Figura 1: Objetivos y estrategias de OCII de IMARPE.

\begin{tabular}{|c|c|c|}
\hline OBJETIVOS & \multicolumn{2}{|c|}{$\begin{array}{l}\text { - Mejorar la comunicación entre la sede central y los laboratorios descentralizados. } \\
\text { - Aplicar técnicas de comunicación interna para los trabajadores a nivel nacional. } \\
\text { - Promover el uso de nuevas TIC. } \\
\text { - Brindar asesoramiento y apoyo técnico en materia de comunicaciones a los laboratorios } \\
\text { - Difundir el manual de identidad corporativa al interior de IMARPE. }\end{array}$} \\
\hline \multirow{4}{*}{ ESTRATEGIAS } & Nuevas TIC & $\begin{array}{l}\text { - Apertura de una canal de YouTube para la emisión de contenido } \\
\text { institucional a nivel nacional. } \\
\text { - Producción de un programa informativo en televisión, cuyo } \\
\text { contenido sea noticias referentes a la organización a nivel nacional e } \\
\text { internacional. } \\
\text { - Uso del Facebook y Twitter para socializar información, controlado y } \\
\text { monitoreado por la OCll de IMARPE. }\end{array}$ \\
\hline & $\begin{array}{l}\text { Gestión del } \\
\text { conocimiento }\end{array}$ & $\begin{array}{l}\text { - Elaboración de boletines internos mensuales. } \\
\text { - Creación de una sección dentro del programa televisivo interno y el } \\
\text { boletín informativo "Hablan las provincias". } \\
\text { - Reuniones mensuales con los jefes de laboratorios descentralizados } \\
\text { para que compartan los proyectos que vienen desarrollando en su } \\
\text { región. }\end{array}$ \\
\hline & $\begin{array}{l}\text { Elaboración } \\
\text { del manual } \\
\text { de identidad } \\
\text { corporativa } \\
\text { (MIC) }\end{array}$ & $\begin{array}{l}\text { Este documento será diseñado y diagramado para el público interno, } \\
\text { usando un lenguaje claro y conciso. } \\
\text { - Será difundido de manera física y virtual a todos los trabajadores. } \\
\text { - Deberá incluir la normativa de uso del logo institucional en versiones: } \\
\text { color, blanco y negro y sepia. } \\
\text { - Inclusión de la línea gráfica institucional (colores: azul, turquesa, } \\
\text { celeste, amarillo). }\end{array}$ \\
\hline & $\begin{array}{l}\text { Trabajar } \\
\text { en red }\end{array}$ & $\begin{array}{l}\text { - La OCII implementará un área dedicada a asesorar a los laboratorios } \\
\text { descentralizados, para la elaboración de material de difusión, } \\
\text { publicidad y posicionamiento. } \\
\text { - Realización de actividades de integración entre el personal para } \\
\text { mejorar el clima laboral. } \\
\text { - Visitas trimestrales por parte de los profesionales de comunicaciones } \\
\text { a los laboratorios descentralizados para evaluar y monitorear el trabajo } \\
\text { de comunicaciones local. }\end{array}$ \\
\hline
\end{tabular}

Fuente: IMARPE (2016). 
IMARPE recibe información actualizada a través de sus laboratorios descentralizados, los mismos que dan cuenta de la realidad marino-ambiental del litoral. Sin embargo, a pesar de la importancia de su trabajo, estos centros de investigación se erigen interdependientes frente a la sede central: algunos de estos no usan el logo institucional oficial, producen su propia línea gráfica y realizan actividades de comunicación sin previa coordinación con la sede del Callao.

Esta aparente desconexión se agudiza por la existencia de tres grupos que integran IMARPE: personal científico (encargados de la organización, investigación, monitoreo, y evaluación de los recursos pesqueros), administrativo (ejecución de presupuesto, mantenimiento de la infraestructura, recursos humanos, logística, etc.) y naval (oficiales y suboficiales de la Marina de Guerra del Perú para el manejo y mantenimiento de los buques de investigación).

Estos grupos poseen diferente formación profesional y demuestran desconocimiento hacia las otras profesiones, lo cual genera conflictos en la comunicación y dificulta la aplicación de técnicas y herramientas de RR. PP. Aparte de esta dimensión social, se suman otros factores que ocasionan problemas en la calidad de las relaciones y el correcto manejo de la comunicación: el factor económico (las sedes descentralizadas poseen presupuesto pero sin orientarlos a temas comunicacionales, disparidad salarial) y político (frecuente rotación de la alta dirección).

\section{Metodología}

La muestra está conformada por 196 trabajadores del IMARPE. Para su selección, se empleó la técnica de muestreo no probabilístico por conveniencia o criterio. La técnica de recolección de datos elegida fue la encuesta. El cuestionario estuvo integrado por 49 ítems. Para determinar la validez del instrumento se utilizó la prueba de juicio de expertos, procesado mediante la fórmula de coeficiente de validez Aiken. Asimismo, para determinar la confiabilidad del instrumento se utilizó el coeficiente Alfa de Crombach.

\section{Resultados}

Se observa que un gran porcentaje de encuestados (95.2\%) manifiestan que las RR. PP. son adecuadas para la construcción, manejo y mantenimiento de la imagen organizacional de IMARPE y la identidad de los colaboradores. El 88.8\% de los encuestados refirieron que el trabajo realizado por los profesionales de comunicaciones (en la aplicación de técnicas y herramientas de las RR. PP.) ha generado una adecuada identidad en los trabajadores de IMARPE.

Respecto a la imagen organizacional de IMARPE, los encuestados manifestaron en su mayoría tener una adecuada imagen organizacional referida a la apreciación de las técnicas de RR. PP. aplicadas en la construcción y mantenimiento de la imagen percibida, real y deseada por los colaboradores. Asimismo, reconocen el prestigio nacional e internacional de su personal técnico-científico, así como también aspiran tener una mejor calidad de relaciones a nivel interno para mejorar la reputación de la organización hacia sus públicos externos. 
Referente a la GC de IMARPE, los encuestados refirieron en un gran porcentaje $(77.7 \%)$ que es adecuada ${ }^{9}$. En cuanto a los flujos de comunicación en IMARPE, los encuestados manifestaron en su mayoría (73.9\%) que son adecuados, debido a que los colaboradores indicaron recibir información acerca de las políticas y metas de la organización, sin dejarse de lado que el $26.1 \%$ de encuestados refirieron que los flujos de la comunicación son inadecuados debido a que la comunicación descendente es la que prima dentro de la organización, debido a que la mayoría de mensajes son emitidos para dar indicaciones $\mathrm{u}$ órdenes.

El 56.9\% de encuestados precisaron que los canales de comunicación en IMARPE, son adecuados, debido a que los dispositivos de comunicación interna (boletines, videos, intranet) emiten información actualizada para los colaboradores. Sin embargo, el $43.1 \%$ de los encuestados refirieron que son inadecuados, puesto que la información llega a través del rumor que es reconocido como un canal de comunicación informal dentro de la organización.

Para determinar la asociación entre las RR. PP. y la GC, utilizamos el coeficiente de Spearman (figura 2), ya que hacemos uso de variables en escala ordinal:

Figura 2: Asociación entre las RR. PP. y la GC en IMARPE.

\begin{tabular}{|l|c|c|}
\hline \multirow{2}{*}{$\begin{array}{c}\text { Relaciones públicas } \\
\text { y gestión de la } \\
\text { comunicación }\end{array}$} & Valor del coeficiente de Spearman & 0.000 \\
\cline { 2 - 3 } & 0.662 & $\begin{array}{c}\text { Probabilidad } \\
\text { (Sig. Aproximada) }\end{array}$ \\
\hline Nota: & 188 \\
Nivel de confianza del $95 \%$ o significancia $\alpha=0.05$ & \\
Las correlaciones son significativas cuando la probabilidad es menor a $\alpha=0.05$ & \\
\hline
\end{tabular}

Fuente: Elaboración propia.

Observamos que la probabilidad (0.000) es menor al valor de significancia $\alpha=0.05$, lo que nos indica que hay significancia estadística y existe asociación significativa entre las RR. PP. y la GC en IMARPE. La influencia entre estas dos variables es moderada (0.662), directa o positiva, que cuando una variable mejora la otra también y viceversa. El $77.1 \%$ de encuestados coincide en que cuando las RR. PP. son adecuadas, la GC también es adecuada. Sin embargo, el 18.1\% de los encuestados coincide que las adecuadas RR. PP. conllevan a una GC inadecuada en IMARPE.

9 Siendo IMARPE un organismo público (cuya comunicación es formal y vertical) se ve obligado a seguir los lineamientos que le indica el Estado Peruano. 
Para determinar la asociación entre identidad de los colaboradores y la GC, utilizamos el coeficiente de Spearman (figura 3) ya que hacemos uso de variables en escala ordinal.

Figura 3: Asociación entre identidad de colaboradores y la GC en IMARPE.

\begin{tabular}{|l|c|c|}
\hline \multirow{2}{*}{$\begin{array}{c}\text { Identidad de los colaboradores y la gestión } \\
\text { de la comunicación }\end{array}$} & $\begin{array}{c}\text { Valor del coeficiente } \\
\text { de Spearman }\end{array}$ & $\begin{array}{c}\text { Probabilidad } \\
\text { (Sig. Aproximada) }\end{array}$ \\
\cline { 2 - 3 } Nota: & 0.568 & 0.000 \\
$\mathrm{~N}=188$ & \\
Nivel de confianza del 95\% o significancia $\alpha=0.05$ & \\
Las correlaciones son significativas cuando la probabilidad es menor a $\alpha=0.05$ & \\
\hline
\end{tabular}

Fuente: Elaboración propia.

Observamos que la probabilidad (0.000) es menor al valor de significancia $\alpha=0.05$, lo que nos indica que hay significancia estadística y existe asociación significativa entre la identidad de los colaboradores y la GC en IMARPE. La influencia entre estas dos variables es moderada (0.568) y directa o positiva, es decir, que cuando una variable mejora la otra también y viceversa. Apreciamos que el mayor porcentaje de coincidencia es del $73.4 \%$ de los encuestados, que cuando refieren una adecuada identidad de los colaboradores, la GC también es adecuada en IMARPE. Identificamos igualmente un considerable porcentaje de encuestados (15.4\%) que manifestaron una adecuada identidad que conlleva a una inadecuada gestión de comunicación.

Para determinar la asociación entre imagen organizacional para los colaboradores y la GC, utilizamos el coeficiente de Spearman (figura 4) ya que hacemos uso de variables en escala ordinal:

Figura 4: Asociación entre imagen organizacional para los colaboradores y la GC en IMARPE.

\begin{tabular}{|c|c|c|}
\hline \multirow{2}{*}{$\begin{array}{c}\text { Imagen organizacional para los } \\
\text { colaboradores y gestión de la } \\
\text { comunicación }\end{array}$} & $\begin{array}{c}\text { Valor del coeficiente de } \\
\text { Spearman }\end{array}$ & $\begin{array}{c}\text { Probabilidad } \\
\text { (Sig. Aproximada) }\end{array}$ \\
\hline & 0.645 & 0.000 \\
\hline \multicolumn{3}{|l|}{ Nota: } \\
\hline \multicolumn{3}{|l|}{$N=188$} \\
\hline \multicolumn{3}{|c|}{ Nivel de confianza del $95 \%$ o significancia $\alpha=0.05$} \\
\hline \multicolumn{3}{|c|}{ Las correlaciones son significativas cuando la probabilidad es menor a $\alpha=0.05$} \\
\hline
\end{tabular}

Fuente: Elaboración propia. 
Observamos que la probabilidad (0.000) es menor al valor de significancia $\alpha=0.05$, lo que nos indica que hay significancia estadística y existe asociación significativa entre la imagen organizacional para los colaboradores y la gestión de la comunicación en IMARPE. La influencia entre estas dos variables es moderada (0.645) y directa o positiva, es decir, que cuando una variable mejora la otra también y viceversa. El mayor porcentaje de coincidencia es del $77.7 \%$ de encuestados, lo cual coincide cuando hay una adecuada imagen organizacional para los colaboradores, la gestión de comunicación igualmente es adecuada. La otra mayor coincidencia es del 19.1\% de los encuestados, quienes concuerdan que una adecuada imagen organizacional para los colaboradores conlleva a una inadecuada gestión de la comunicación en IMARPE.

Para determinar la asociación entre la identidad de los colaboradores y los flujos de comunicación, utilizamos el coeficiente de Spearman (figura 5), ya que hacemos uso de variables en escala ordinal:

Figura 5: Asociación entre la identidad de los colaboradores y los flujos de comunicación en IMARPE.

\begin{tabular}{|l|c|c|}
\hline \multirow{2}{*}{ Identidad y los flujos de la comunicación } & $\begin{array}{c}\text { Valor del coeficiente de } \\
\text { Spearman }\end{array}$ & $\begin{array}{c}\text { Probabilidad } \\
\text { (Sig. Aproximada) }\end{array}$ \\
\cline { 2 - 3 } & 0.658 & 0.000 \\
Nota: & \\
$N=188$ & \\
Nivel de confianza del 95\% o significancia $\alpha=0.05$ & \\
Las correlaciones son significativas cuando la probabilidad es menor a $\alpha=0.05$
\end{tabular}

Fuente: Elaboración propia.

Observamos que la probabilidad (0.000) es menor al valor de significancia $\alpha=0.05$, lo que nos indica que hay significancia estadística y, por lo tanto, existe asociación significativa entre la identidad de los colaboradores y los flujos de la comunicación en IMARPE. La influencia entre estas dos variables es moderada (0.658), directa o positiva, es decir, que cuando una variable mejora la otra también y viceversa. Un gran porcentaje de encuestados refieren que cuando hay una adecuada identidad de los colaboradores, los flujos de comunicación igualmente son adecuados. El 18.6\% de los encuestados concuerdan que cuando la identidad de los colaboradores es adecuada, los flujos de comunicación son inadecuados en IMARPE.

Para determinar la asociación entre la imagen organizacional para los colaboradores y los flujos de comunicación, utilizamos el coeficiente de Spearman (figura 6) ya que hacemos uso de variables en escala ordinal: 
Figura 6: Asociación entre la imagen organizacional para los colaboradores y los flujos de comunicación en IMARPE.

\begin{tabular}{|l|c|c|}
\hline \multirow{2}{*}{$\begin{array}{c}\text { Imagen organizacional para los colaboradores } \\
\text { y los flujos de la comunicación }\end{array}$} & $\begin{array}{c}\text { Valor del coeficiente de } \\
\text { Spearman }\end{array}$ & $\begin{array}{c}\text { Probabilidad } \\
\text { (Sig. Aproximada) }\end{array}$ \\
\cline { 2 - 3 } & 0.660 & 0.000 \\
\hline Nota: & 188 \\
Nivel de confianza del 95\% o significancia $\alpha=0.05$ & \\
Las correlaciones son significativas cuando la probabilidad es menor a $\alpha=0.05$ & \\
\hline
\end{tabular}

Fuente: Elaboración propia.

Observamos que la probabilidad (0.000) es menor al valor de significancia $\alpha=0.05$, lo que nos indica que hay significancia estadística por lo tanto existe asociación significativa entre la imagen organizacional para los colaboradores y los flujos de la comunicación en el Instituto del Mar del Perú-IMARPE. La influencia entre estas dos variables es moderada (0.660) y directa o positiva es decir que cuando una variable mejora la otra también y viceversa. Apreciamos que el mayor porcentaje de coincidencia es del $73.9 \%$ de encuestados que refieren una adecuada imagen organizacional para los colaboradores conlleva a un adecuado flujos de comunicación. Mientras que un $22.9 \%$ de encuestados que refieren una adecuada imagen organizacional para los colaboradores, los flujos de comunicación son inadecuados en el Instituto del Mar del Perú-IMARPE.

Para determinar la asociación entre la identidad de los colaboradores y los canales de comunicación, utilizamos el coeficiente de Spearman (figura 7), ya que hacemos uso de variables en escala ordinal:

Figura 7: Asociación entre la identidad de los colaboradores y los canales de comunicación en IMARPE.

\begin{tabular}{|l|c|c|}
\hline \multirow{2}{*}{$\begin{array}{l}\text { Identidad de los colaboradores y canales de la } \\
\text { comunicación }\end{array}$} & $\begin{array}{c}\text { Valor del coeficiente de } \\
\text { Spearman }\end{array}$ & $\begin{array}{c}\text { Probabilidad } \\
\text { (Sig. Aproximada) }\end{array}$ \\
\cline { 2 - 3 } Nota: & 0.132 & 0.071 \\
$\mathrm{~N}=188$ & \\
Nivel de confianza del $95 \%$ o significancia $\alpha=0.05$ & \\
Las correlaciones son significativas cuando la probabilidad es menor a $\alpha=0.05$ & \\
\hline
\end{tabular}

Fuente: Elaboración propia. 
Observamos que la probabilidad (0.071) es mayor al valor de significancia $\alpha=0.05$, lo que nos indica que no hay significancia estadística y, por lo tanto, no hay asociación significativa entre la identidad de los colaboradores y los canales de comunicación en IMARPE. La influencia entre estas dos variables es muy débil (0.132), pero directa o positiva, es decir, que cuando una variable mejora la otra también y viceversa. Apreciamos un $52.1 \%$ de encuestados que coinciden en que una adecuada identidad de los colaboradores conlleva a adecuados canales de comunicación en IMARPE; mientras que un 36.7\% de encuestados manifiestan que una adecuada identidad de los colaboradores conlleva inadecuados canales de comunicación.

Para determinar la asociación entre la imagen organizacional para los colaboradores y los canales de comunicación, utilizamos el coeficiente de Spearman (figura 8) ya que hacemos uso de variables en escala ordinal:

Figura 8: Asociación entre la imagen organizacional para los colaboradores y los canales de comunicación en IMARPE.

\begin{tabular}{|l|l|l|}
\hline \multirow{2}{*}{$\begin{array}{l}\text { Imagen organizacional para los } \\
\text { colaboradores y canales de comunicación }\end{array}$} & \multicolumn{1}{|c|}{$\begin{array}{c}\text { Valor del coeficiente de } \\
\text { Spearman }\end{array}$} & $\begin{array}{c}\text { Probabilidad } \\
\text { (Sig. Aproximada) }\end{array}$ \\
\cline { 2 - 3 } Nota: & 0.233 & 0.001 \\
$\mathrm{~N}=188$ & \\
Nivel de confianza del $95 \%$ o significancia $\alpha=0.05$ & \\
Las correlaciones son significativas cuando la probabilidad es menor a $\alpha=0.05$
\end{tabular}

Fuente: Elaboración propia.

Observamos que la probabilidad (0.001) es menor al valor de significancia $\alpha=0.05$, lo que nos indica que hay significancia estadística y, por lo tanto, existe asociación significativa entre la imagen organizacional para los colaboradores y los canales de comunicación en IMARPE. La influencia entre estas dos variables es débil (0.233), pero directa o positiva, es decir, que cuando una variable mejora la otra también y viceversa.

Observamos que un $56.4 \%$ de los encuestados coinciden en que una adecuada imagen organizacional para los colaboradores conlleva a adecuados canales de comunicación en IMARPE; mientras que un $40.4 \%$ de encuestados manifiestan que una adecuada imagen organizacional para los colaboradores conlleva a inadecuados canales de comunicación.

\section{Conclusiones y recomendaciones}

En las diferentes dimensiones de las RR. PP. y la GC, observamos que la mayoría alcanzaron coeficientes de correlación significativos, dándonos como resultado una relación directa 
entre ambas variables. Cabe resaltar que no existe asociación significativa entre las dimensiones "identidad" y "canales de la comunicación", debido a que la probabilidad (0.071) es mayor al valor de significancia.

El análisis de las correlaciones entre los puntajes de las escalas de las dimensiones "imagen e identidad" (de la variable RR. PP.) y las dimensiones "flujos de la comunicación y canales de la comunicación" (de la variable GC) indican que se alcanzaron correlaciones estadísticas significativas y positivas en todos los casos. Al analizarse los resultados obtenidos podemos afirmar que la hipótesis general de investigación (existe relación significativa entre las RR. PP. y la GC) ha sido validada.

Se sugiere la aplicación de técnicas de comunicación interna para mantener y elevar el nivel de identidad de los trabajadores, lo que conllevará a una proyección de imagen positiva de la organización, ya que es el público interno el portador primario de imagen. Asimismo, se recomienda el uso de nuevas TIC con el fin de mantener informado al público interno y mejorar la calidad de relaciones entre los trabajadores. Del mismo modo, se sugiere impulsar la elaboración de un manual de identidad corporativa que ayudará a conocer la línea gráfica de la organización a los trabajadores.

Asimismo, se propone la promoción de la comunicación horizontal dentro de la organización, a través de reuniones mensuales con jefes y líderes de las diferentes dependencias. En ese sentido, se sugiere que la alta dirección de IMARPE realice conversatorios con las diferentes dependencias de la organización, no sólo con los de la sede central, sino con los laboratorios descentralizados, para mejorar la imagen de la institución.

Se recomienda la elaboración de planes de comunicación estratégica que minimicen la existencia de rumores para promover la relación entre la identidad y los canales de comunicación. Por último, se plantea el uso de canales de comunicación formal, no sólo para emitir órdenes o encargar tareas, sino para promover, felicitar y hacer participar a los trabajadores del proceso de comunicación dentro de la organización.

\section{Fuentes consultadas}

Andrade, M. (2014). Las Relaciones Públicas y la Gestión de la Comunicación en tres institutos especializados del Ministerio de Salud 2013 [tesis de maestría]. Lima: Universidad de San Martín de Porres, Facultad de Ciencias de la Comunicación, Turismo y Psicología.

Ayala, M. (2011). Relaciones Públicas y su concreción teórica y funcional como disciplina independiente. Análisis del ámbito latinoamericano [tesis de doctorado]. Lima: Universidad de San Martín de Porres, Facultad de Ciencias de la Comunicación, Turismo y Psicología.

Brandolini, A. y González Frígoli, M. (2009). Comunicación interna. Recomendaciones y errores frecuentes. Buenos Aires: La Crujía Ediciones. 
Castillo, A. (2010). Introducción a las Relaciones Públicas. Málaga: Instituto de Investigación en Relaciones Públicas. Extraída el 9/V/2016 desde http://webpersonal.uma.es/DE/ACASTILLOE/librorelacionespublicas.pdf

\section{Costa, J.}

(2009a). El Dircom hoy. Dirección y gestión de la comunicación en la nueva economía. Barcelona: Costa Punto Com.

_(2009b). Imagen corporativa en el siglo XXI. Buenos Aires: La Crujía Ediciones.

Diez, S. (2011). Técnicas de comunicación. La comunicación en la empresa. Bogotá: Ediciones de la U.

Elías, L. (2014). Relación entre la comunicación interna y la administración de la controversia pública en los colaboradores de la gerencia de desarrollo social y lucha contra la pobreza de la Municipalidad Distrital de Villa María del Triunfo, mayo-junio 2014 [tesis de maestría]. Lima, Universidad de San Martín de Porres, Facultad de Ciencias de la Comunicación, Turismo y Psicología.

\section{Ferrari, M.}

_(2012). "Comunicación y Cultura: análisis de la realidad de las Relaciones Públicas en organizaciones chilenas y brasileñas". Cuadernos del Centro de Estudios en Diseño y Comunicación. Vol. IV, núm. 40, pp. 47-64.

_(2004). Relaciones Públicas y comunicación en Latinoamérica. Conferencia Latinoamericana. Córdoba: IPRA.

Ferrari, M. y França, F. (2011). Relaciones públicas. Naturaleza, función y gestión en las organizaciones contemporáneas. Buenos Aires: La Crujia Ediciones.

Flores, F. (1999). Relaciones Públicas Año 2000. Lima: CONCYTEC.

Grunig, J.; Ferrari, M. y França, F. (2009). Relaciones Públicas. Teoría, contexto y relacionamientos. Barcelona: Gestión 2000.

Grunig, J.; Serra, G.; Noguero, A. y Solórzano, E. (2010). Las nuevas Relaciones Públicas. Lima: Universidad de San Martín de Porres.

Harrison, S. (2002). Relaciones Públicas. Una introducción. Madrid: Thomson.

IMARPE, Instituto del Mar del Perú (2016). “Antecedentes, misión, visión, organigrama, ROF y MOF”. Extraída el 9/V/2016 desde www.imarpe.gob.pe

León, R. (2014). El impacto de las Relaciones Públicas en los servicios de bienestar universitario en relación a las actitudes y sentido de pertenencia de los alumnos de la Escuela de Ciencias de la Comunicación, USMP 2013 [tesis de maestría]. Lima: Universidad de San Martín de Porres, Facultad de Ciencias de la Comunicación, Turismo y Psicología. 
Losada, J. (2004). Gestión de la comunicación en las organizaciones. Barcelona: Ariel.

Marston, J. (1988). Relaciones Públicas modernas. México: McGraw-Hill.

Matilla, K. (2009). Conceptos fundamentales en la planificación estratégica de las Relaciones Públicas. Barcelona: UOC.

Morales, F. (2006). Comunicación Planificada. Estudio cualitativo de las variables estructura, gestión y valores en la comunicación de las organizaciones [tesis de doctorado]. Barcelona: Universidad Autónoma de Barcelona. Extraída el 9/V/2016 desde http://www.tdx.cat/handle/10803/4131

Ortiz, J. (2014). 50 años de mar y ciencia. Lima: Instituto del Mar del Perú.

Pérez, R. y Solórzano, E. (1999). Relaciones Públicas Superiores. Una nueva pedagogía. Lima: Universidad de San Martín de Porres.

Pinto, P. y Montenegro, P. (2013). Plan estratégico de comunicación interna para la empresa de Catering Service del Grupo Hanaska. Quito: Universidad de las Américas.

Publicometa.info (2007, septiembre 27). "Entrevista al prof. Román Pérez Senac". Extraída el 9/V/2016 desde

http://publicometa.fullblog.com.ar/entrevista_al_prof_roman_perez_ senac_351190900515.html

Solá, F. (2013). Las Relaciones Públicas en la estrategia de comunicación de la Guardia Civil de Alicante: aplicación de modelos conductuales [tesis de doctorado]. Alicante: Universidad de Alicante. Extraída el 9/V/2016 desde

http://rua.ua.es/dspace/bitstream/10045/35863/1/Tesis_lorenzo_sola.pdf

Solano, L. (1995). Fundamentos de las Relaciones Públicas. Madrid: Síntesis.

Solórzano, E. (2009). “Teoría y Práctica de las Relaciones Públicas en el Perú”. Revista Cientifica de la Asociación Latinoamericana de Carreras de Relaciones Públicas, ALACAURP, núm. 5, pp. 111-134.

Spell, C. (2013). Categorías y roles de excelencia en una firma consultora de Relaciones Públicas [tesis de maestría]. Lima: Universidad de San Martín de Porres, Facultad de Ciencias de la Comunicación, Turismo y Psicología.

Stephenson, H. (1960). Relaciones Públicas. Lima: PUCP.

Strauck, M. y Guillén, C. (2014). "La gestión de la comunicación en los gobiernos locales, una mirada desde la perspectiva estratégica: análisis del caso peruano". Revista Internacional de Relaciones Públicas. Vol. IV, núm. 7, pp. 153-176. 
Villar, M. (2013). Sistema para el diagnóstico de la comunicación interna caso de estudio Instituto Nacional del Niño y la Familia [tesis de doctorado]. Barcelona: Universidad Politécnica de Cataluña. Extraída el 9/V/2016 desde http://www.tdx.cat/ handle/10803/277425

Wilcox, D.; Cameron, G. y Xifra, J. (2006). Relaciones Públicas, estrategias y tácticas. Madrid: Pearson.

\section{Xifra, J.}

_(2006). "Lucien Matrat y la consolidación de las relaciones públicas en Europa”. Extraída el 9/V/2016 desde http://www.google.com.pe/url?sa=t\&rct=j\&q=\&esrc=s\&source=web\&cd=1\&ved=0CC4 QFjAA\&url=http $\% 3 \mathrm{~A} \% 2 \mathrm{~F} \% 2 \mathrm{Frevistas} . \mathrm{ucm} . \mathrm{es} \% 2 \mathrm{Findex} . \mathrm{php} \% 2 \mathrm{FHICS} \% 2 \mathrm{Farticle} \% 2 \mathrm{Fd}$ ownload\%2FHICS0606110229A\%2F19184\&ei=zLM0UfXPApTo9gTQ1oCgCg\&usg=A FQjCNGyeooMahuw7SsLMXrmxSJhpfqhNg\&bvm=bv.4314 _(2003). Teoría y estructura de las Relaciones Públicas. Madrid: McGraw-Hill. 